\title{
An Equation for Moist Entropy in a Precipitating and Icy Atmosphere
}

\author{
Xiping Zeng \\ Goddard Earth Sciences and Technology Center, University of Maryland, Baltimore County, \\ and Laboratory for Atmospheres, NASA Goddard Space Flight Center, Greenbelt, Maryland \\ Wei-Kuo Tao and Joanne Simpson \\ Laboratory for Atmospheres, NASA Goddard Space Flight Center, Greenbelt, Maryland
}

\section{Popular Summary}

Moist entropy is nearly conserved in adiabatic motion. It is redistributed rather than created by moist convection. Thus moist entropy and its equation, as a healthy direction, can be used to construct analytical and numerical models for the interaction between tropical convective clouds and large-scale circulations. Hence, an accurate equation of moist entropy is needed for the analysis and modeling of atmospheric convective clouds.

On the basis of the consistency between the energy and the entropy equations, a complete equation of moist entropy is derived from the energy equation. The equation expresses explicitly the internal and external sources of moist entropy, including those in relation to the microphysics of clouds and precipitation. In addition, an accurate formula for the surface flux of moist entropy from the underlying surface into the air above is derived.

Because moist entropy deals "easily" with the transition among three water phases, it will be used as a prognostic variable in the next generation of cloud-resolving models (e. g. a global cloud-resolving model) for low computational noise. Its equation that is derived in this paper is accurate and complete, providing a theoretical basis for using moist entropy as a prognostic variable in the long-term modeling of clouds and large-scale circulations. 


\title{
An Equation for Moist Entropy in a Precipitating and Icy
}

\section{Atmosphere}

\author{
Xiping Zeng* \\ Goddard Earth Sciences and Technology Center, University of Maryland, Baltimore \\ County, and Laboratory for Atmospheres, NASA Goddard Space Flight Center, \\ Greenbelt, Maryland
}

Wei-Kuo Tao and Joanne Simpson

Laboratory for Atmospheres, NASA Goddard Space Flight Center, Greenbelt, Maryland

September 10, 2003

Submitted to Journal of the Atmospheric Sciences

Corresponding author address: Dr. Xiping Zeng, Mail Code 912, Rm A417, Bldg 33, NASA/Goddard Space Flight Center, Greenbelt, MD 20771. Email: zeng@agnes.gsfc.nasa.gov 


\begin{abstract}
This paper addresses an equation for moist entropy in the framework of cloudresolving models. Since the energy equation is equivalent to the entropy equation with proper source terms, this paper for the first time derives a complete equation for moist entropy from the energy equation. The equation expresses the internal and external sources of moist entropy explicitly, providing a basis for moist entropy as a prognostic variable in long-term cloud modeling. In addition, an accurate formula for the surface flux of moist entropy from the underlying surface into the air above is derived.

Moist entropy is used to rewrite the Neelin-Held model for the diagnosis of large-scale vertical velocity. After applying the model to a tropical oceanic atmosphere with mean annual soundings, the paper shows the sensitivity of large-scale vertical circulations to the radiative cooling rate and the surface flux of moist entropy, which demonstrates the necessity for an accurate equation for moist entropy in the analysis and modeling of large-scale tropical circulations.
\end{abstract}




\section{Introduction}

The motive for an accurate equation for moist entropy has two origins: numerical modeling and theoretical analysis of tropical clouds and their interaction with large-scale circulations. On one side, the motivation comes from the rapid development of massive parallel computation. With the great increase in computational power, cloud-resolving models have significantly increased their horizontal domain size. In the near future, the domain size will reach thousands of kilometers so that the models can accommodate both clouds and large-scale circulations. With the explicit representation of clouds and largescale circulations, the models can be used to explore the interaction between clouds and large-scale circulations. Since the models with large-scale circulations usually integrate for days or longer, they should represent the energy equation accurately and reduce computational noise, especially that from the connection between microphysical and dynamic processes.

Most current cloud-resolving models use three prognostic thermodynamic variables to simulate non-precipitating warm clouds and those three variables plus others to simulate other clouds (e.g., Grabowski 1989, Tao and Simpson 1993, Tompkins and Craig 1998, Tao et al. 2003). The three prognostic variables are temperature (or its equivalent) and the mixing ratios of water vapor and cloud water. In models with the three prognostic variables, there are computational phenomena such as spurious supersaturation near cloud edges (e.g., Grabowski 1989). Special adjustment techniques are usually introduced to reduce their negative effects, benefiting the numerical simulation of individual cloud systems (e.g., Tao et al. 1989). 
Some computational phenomena originate in the choice of prognostic thermodynamic variables (Grabowski 1989, Ooyama 1990, Zeng 2001). In a new trend to avoid them, moist entropy is used as a prognostic variable in modeling, and temperature is diagnosed from that and other prognostic variables (Raymond and Blyth 1986; Ooyama 1990, 2001; Zeng 2001). Raymond and Blyth (1986) used moist entropy and the total mixing ratio of airborne water (water vapor and cloud water) as prognostic variables in a parcel model. Ooyama (1990) analyzed the thermal relations of moist entropy and suggested moist entropy be used as a prognostic variable in multiple-dimensional numerical models. Ooyama (2001) and Zeng (2001) constructed two- and three-dimensional models with moist entropy as a prognostic variable, respectively, to simulate warm clouds. In their models neither spurious supersaturation nor negative mixing ratio of cloud water appears. Since moist entropy deals with water phase transition "easily", it is logical to extend moist entropy as a prognostic variable to simulate cold clouds. Hence, an accurate equation for moist entropy is needed in the modeling and analysis of cold clouds.

However some researchers, on the basis of the consistency between the entropy and the energy equations, have questioned the accuracy of moist entropy as a prognostic variable in long integration (Satoh 2002). In fact, the entropy equation is consistent with the energy equation if the sources of moist entropy (including irreversible generation) are expressed accurately. This paper addresses the question on the consistency between the energy and the entropy equations and derives accurate source terms for moist entropy from the energy equation.

Likewise, there is motivation for an accurate equation for moist entropy with regard to research on the interaction between tropical convection and large-scale circulations. In 
the tropics, convective forcing is thermodynamic rather than dynamic. On sufficiently small time- and space-scales, dynamic forcing (e.g., lifting to the level of free convection) is important. However, convection is self-limiting. Sustaining convective forcing depends upon the maintenance of favorable thermodynamic conditions. As a result, thermodynamics dominate on sufficiently large scales (Raymond 1995, 2000; Raymond and Zeng 2000). Therefore, it is imperative to represent thermodynamics accurately in studying the interaction between tropical convection and large-scale circulations.

However, not all variables representing the thermodynamics approach the interaction straightforwardly. For example, Charney and Eliassen (1964) used potential temperature to represent the energy equation to study the growth of a hurricane depression. After connecting the convective heating rate to the low-level convergence of water vapor, they introduced a concept of convective instability of the second kind. The concept is specious. It is questioned with moist entropy (or equivalent potential temperature) as follows. Assuming that the surface moist entropy is fixed and horizontally uniform, the midtropospheric temperature never surpasses the temperature of an air parcel that moves adiabatically from the surface to the middle troposphere, regardless of how large the lowlevel convergence is. In other words, there is an upper limit on the mid-tropospheric temperature. However, the upper limit is contrary to the prediction of temperature by the linear model of Charney and Eliassen.

In contrast to (potential) temperature, moist entropy is suitable for representing the thermodynamics involved in the interaction between tropical convection and large-scale circulations. Moist entropy is nearly conserved in adiabatic motion. Thus moist entropy is redistributed rather than created by moist convection. If a conceptual model is based on 
the entropy equation, it can avoid implicit assumptions that address specious concepts such as convective instability of the second kind. Neelin and Held (1987) constructed a simple model of moist static energy budget, providing a starting point for understanding the large-scale vertical circulation in the tropics. This paper rewrites their model on the basis of a moist entropy equation, incorporating parcel dynamics reasoning. From the model, it is impossible to reach the concept of Charney and Eliassen (1964).

Moist entropy plays a unique role in the analysis of tropical convection. Thus, its accurate equation is needed in the modeling and analysis of the interaction between tropical clouds and large-scale circulations. However, for the time being, there is no accurate equation for moist entropy in a precipitating and icy atmosphere. This paper derives such an equation for moist entropy from the energy equation.

The paper consists of six sections. Section 2 explains the consistency between the energy and the entropy equations. Section 3 derives an expression for moist entropy with cloud ice. Section 4 expresses the sources of moist entropy, including those in relation to the microphysics of clouds and precipitation. Section 5 presents a flux-form equation for moist entropy and derives an accurate formula for the surface flux of moist entropy from the underlying surface into the air above. And finally, Section 6 gives a summary and discusses future work. Except for special illustrations, the paper follows the symbol definitions in Appendix A.

\section{Consistency between the entropy and the energy equations}

This section explains the consistency between the entropy and the energy equations. Entropy $s$ changes when a body is in thermal contact with its environment $\left(d_{e} s\right)$ or 
increases as a result of internal changes within the body $\left(d_{i} s\right)$. Thus, the total change in the entropy of a body is given by (Wallace and Hobbs 1977)

$$
d s=d_{e} s+d_{i} s
$$

The change $d_{e} s$ is given by

$$
d_{e} s=\frac{d q}{T}
$$

The second law of thermodynamics states

$$
d_{i} s \geq 0
$$

Combining Equations (2.1) and (2.2) gives

$$
d s=T^{-1} d q+d_{i} s
$$

Although Equation (2.3) shows the sign of $d_{i} s$, it can't complete Equation (2.4) for a solution. The first law of thermodynamics deals with energy conservation, connecting $d q$ to $d T$ and $d p$. After replacing $d q$ in Equation (2.4) with that in the first law of thermodynamics, the expression for $d_{i} s$ is obtained in terms of the thermodynamic properties $T, p$ and $s$. In other words, the entropy equation (2.4) is equivalent to the energy equation if $d_{i} s$ is represented correctly.

On the above principle, $d_{i} s$ can be determined with the aid of the energy equation while the expression for entropy is known. Consider a dry atmosphere without diabatic cooling, for example, its potential temperature and entropy are

$$
\begin{aligned}
& \theta=T\left(p_{r e f} / p\right)^{R_{d} / C_{p}} \\
& s_{d}=C_{p} \ln \left(\theta / T_{r e f}\right)
\end{aligned}
$$

respectively (Iribarne and Godson 1981). Its energy equation is

$$
\rho d \theta / d t=\nabla \cdot\left(\rho K_{h} \nabla \theta\right)
$$


where $K_{h}$ is the eddy mixing coefficient for scalars. Dividing both sides of the preceding equation by $\theta$ and rearranging the right side yields

$$
\rho d \ln \theta / d t=\nabla \cdot\left(\rho K_{h} \nabla \ln \theta\right)+\rho K_{h} \nabla \ln \theta \cdot \nabla \ln \theta
$$

or

$$
\rho d s_{d} / d t=\nabla \cdot\left(\rho K_{h} \nabla s_{d}\right)+\rho C_{p}^{-1} K_{h} \nabla s_{d} \cdot \nabla s_{d}
$$

where the last term expresses the irreversible generation of entropy due to mixing.

In a dry atmosphere, heat passes from warm to cold areas, just as the second law of thermodynamics describes. The heat flux from warm to cold areas is expressed quantitatively by an empirical formula or the last term in Equation (2.7). It is the empirical formula that leads to the expression for $d_{i} s$. Therefore, the empirical formula contains more quantitative information than the second law of thermodynamics.

\section{Moist entropy}

\section{a. Moist entropy and equilibrium}

The concept of entropy is now clear (e.g., Morse 1969). It is discussed briefly here to understand different expressions for moist entropy (Betts 1973, Iribarne and Godson 1981, Raymond and Blyth 1986, Hauf and Höller 1987, Ooyama 1990, Emanuel 1994, Zeng 2001). A unique entropy expression makes sense only when an air parcel remains at an equilibrium state. However, an air parcel with three phases of water substance usually stays at a non-equilibrium state because saturation vapor pressure around water and ice particles varies with the radius and phase of the particles (Pruppacher and Klett 1978) and precipitating particles fall relatively with respect to air. As a result, different expressions for moist entropy are introduced for specific applications. 
Moist entropy can be defined as the sum of its constituent entropies plus mixing terms or be introduced on the basis of entropy conservation in adiabatic motion. These two definitions are equivalent when an air parcel stays at an equilibrium state but are slightly different when a parcel stays at a non-equilibrium state. The latter definition is more suitable for cloud modeling and is taken in this paper.

In fact, the issue with moist entropy in meteorology is moist entropy budget (Neelin and Held 1987; Emanuel 1995; Raymond 1995, 2000; Raymond and Zeng 2000) rather than the theoretical definition of moist entropy. Exactly speaking, the issue is the expressions on the internal and external sources of moist entropy while the expression for moist entropy is given. The next subsection derives an expression for moist entropy on the basis of entropy conservation in adiabatic motion. With the expression, Section 4 derives the source terms for moist entropy from the energy equation.

\section{b. An expression for moist entropy}

Consider an air parcel that is in a reversible process and is thermally insulated from its environment. Its moist entropy is conserved. From the energy equation, variables can be found that are conserved. Obviously these conserved variables are either moist entropy or its relatives. In this way, an expression for moist entropy is obtained.

In the approach for an expression for moist entropy, Kirchhoff's relations provide a starting point:

$$
\begin{aligned}
& d L_{v} / d T=C_{p v}-c_{l} \\
& d L_{s} / d T=C_{p v}-c_{i} \\
& d L_{f} / d T=c_{l}-c_{i}
\end{aligned}
$$


where $L_{v}, L_{s}$ and $L_{f}$ are the latent heats of water vaporization, ice sublimation and water freezing, respectively; the constants $C_{p v}, c_{l}$ and $c_{i}$ are the specific heats of water vapor, liquid and ice, respectively. The mixing ratio of water vapor $q_{v}$ is related to the partial pressure of water vapor $e$ by

$$
q_{v}=\frac{R_{d}}{R_{v}} \frac{e}{p-e}
$$

Consider a water-saturated air parcel that is in adiabatic motion and contains no precipitating particles. From its energy equation

$$
\begin{aligned}
& \left(C_{p}+C_{p v} q_{v s w}+c_{l}\left(q_{t}-q_{v s w}-q_{i}\right)+c_{i} q_{i}\right) d T \\
& -R_{d} T d \ln \left(p-E_{s w}\right)-q_{v s w} R_{v} T d \ln E_{s w}=L_{f} d q_{i}-L_{v} d q_{v s w}
\end{aligned}
$$

an expression for moist entropy is derived. Using Equation (3.1) and the ClausiusClapeyron equation

$$
d \ln E_{s w} / d T=L_{v} / R_{v} T^{2}
$$

Equation (3.3) is rearranged to

$$
d\left(\left(C_{p}+c_{l} q_{t}\right) \ln \frac{T}{T_{r e f}}-R_{d} \ln \frac{p-E_{s w}}{p_{\text {ref }}}+\frac{L_{v} q_{v s w}}{T}-\frac{L_{f} q_{i}}{T}\right)=c_{l}\left(\ln \frac{T}{T_{r e f}}\right) d q_{t}+\frac{L_{f} q_{i}}{T^{2}} d T
$$

where $T_{\text {ref }}=273.15 \mathrm{~K}$ and $p_{\text {ref }}=10^{5} \mathrm{pa}$ are the reference temperature and pressure, respectively. From Equation (3.5), the moist entropy per unit mass of dry air ${ }^{1}$ is defined as

\footnotetext{
${ }^{1}$ Moist entropy in Equation (3.6) excludes the contribution from precipitating particles. It represents only the contribution from moist air that consists of dry air, water vapor, cloud water and ice. When cloud water and ice convert to precipitation, moist entropy passes from moist air to precipitating particles correspondingly. For simplicity, the transfer of moist entropy from moist
} 


$$
s=\left(C_{p}+c_{l} q_{t}\right) \ln \frac{T}{T_{\text {ref }}}-R_{d} \ln \frac{p-e}{p_{\text {ref }}}+\frac{L_{v}\left(T_{l}\right)}{T_{l}} q_{v}-\frac{L_{f}(T)}{T} q_{i}
$$

where $T_{l}$ is simply the air temperature for water-saturated air and is the air temperature at the lifting condensation level for water-unsaturated parcels that satisfies (see Section 4.b)

$$
\left(C_{p}+C_{p v} q_{v}\right) \ln \left(T / T_{l}\right)=\left(R_{d}+R_{v} q_{v}\right) \ln \left(e / E_{s w}\left(T_{l}\right)\right)
$$

Using the expression for moist entropy in Equation (3.6), Equation (3.5) is rewritten as

$$
\frac{d s}{d t}=c_{l} \frac{d q_{t}}{d t} \ln \frac{T}{T_{r e f}}+\frac{L_{f} q_{i}}{T^{2}} \frac{d T}{d t}
$$

When neither precipitating particles nor cloud ice exists, moist entropy is conserved in adiabatic motion. The expression for moist entropy in Equation (3.6) is consistent with that of Betts (1973), Emanuel (1994) and Zeng (2001).

\section{An equation for moist entropy}

After the expression for moist entropy in Equation (3.6) is specified, the source terms for moist entropy are derived from the energy equation. To avoid the debate on moist

air to precipitating particles is treated as an internal sink of moist entropy in contrast to external sources such as the radiative sink of moist entropy.

The entropy of water in Equation (3.6a) is related to $T_{\text {ref. }}$. Thus the transfer of moist entropy from moist air to precipitating particles depends upon $T_{\text {ref. }}$. When $T_{\text {ref }}=273.15 \mathrm{~K}$, water entropy is small. As a result, the internal sink of moist entropy in a precipitating atmosphere is small. Its magnitude, as Zeng (2001) showed in his numerical model of warm clouds, is much smaller than that of the radiative sink for moist entropy. 
entropy definition, $s$ in Equation (3.6) can be defined as a "new" variable in any name that readers favor, and the "new" variable is used to rewrite the energy equation next.

Let $C_{t}$ represent the total conversion of cloud water and ice to precipitation and $E_{t}, F_{t}$ and $S_{t}$ the total evaporation, freezing/melting, sublimation/deposition of precipitating particles in an air parcel, respectively. The total mixing ratio of airborne water (water vapor, cloud water and ice) then changes as

$$
d q_{t} / d t=E_{t}+S_{t}-C_{t}
$$

Let $S_{i}$ represent the sublimation of cloud ice or the deposition of water vapor on cloud ice and $F_{i}$ the freezing of cloud water or the melting of cloud ice. When air is unsaturated with respect to water, the mixing ratio of water vapor changes as

$$
d q_{v} / d t=E_{t}+S_{t}+S_{i}-F_{i}
$$

where $-F_{i}$ represents the melting of cloud ice to cloud water, and cloud water evaporates immediately in an unsaturated environment once cloud ice melts.

\section{a. Sources of moist entropy in a water-saturated air parcel}

This subsection derives the source terms for moist entropy from the energy equation when air is saturated with respect to water. A water-saturated air parcel satisfies the following energy equation

$$
\begin{aligned}
& \left(C_{p}+C_{p v} q_{v s w}+c_{l}\left(q_{t}-q_{v s w}-q_{i}+q_{r}\right)+c_{i}\left(q_{i}+q_{s}+q_{g}\right)\right) d T \\
& -R_{d} T d \ln \left(p-E_{s w}\right)-q_{v s w} R_{v} T d \ln E_{s w} \\
& =L_{f}\left(F_{i}+F_{t}-S_{i}\right) d t-L_{s} S_{t} d t-L_{v} d q_{v s w}+Q d t-Q_{p} d t
\end{aligned}
$$

where $Q$ is the rate of diabatic heating and $Q_{p}$ the heat transfer from air to precipitating particles. The latter is introduced so that the precipitating particles keep the same temperature as the air (see Appendix $\mathrm{C}$ for details). Please note that $-d q_{v s w}$ in Equation 
(4.3) represents the conversion of water vapor to both cloud water and ice, and $-S_{i}$ represents the deposition of water vapor on cloud ice. When cloud water exists, $-S_{i}$ measures the Bergeron process in cold clouds.

Differentiating Equation (3.6a), then multiplying by $T$ and combining with Equation (4.3) yields

$$
\frac{d s}{d t}=\frac{L_{f} F_{t}-L_{s} S_{t}+Q-Q_{p}}{T}+c_{l} \ln \frac{T}{T_{\text {ref }}} \frac{d q_{t}}{d t}+\left(\frac{L_{f} q_{i}}{T}-\left(c_{l} q_{r}+c_{i}\left(q_{s}+q_{g}\right)\right)\right) \frac{d T}{T d t}
$$

with the aid of Equations (3.1) and (3.4). Since the term with $d T / d t$ is small, that term is calculated with an approximate value for $d T / d t$ that is estimated from Equation (4.3). Neglecting the small terms in Equation (4.3) and using Equations (3.2) and (3.4) results in the approximation

$$
\begin{aligned}
& \frac{d T}{d t} \approx\left(L_{f}\left(F_{i}+F_{t}-S_{i}\right)-L_{s} S_{t}+Q+\left(L_{\nu} q_{v s w}+R_{d} T\right) \frac{d \ln p}{d t}\right) \\
& \left(C_{p}+C_{p v} q^{*}+\frac{L_{v}^{2} q_{v s w}}{R_{v} T^{2}}\right)^{-1}
\end{aligned}
$$

From the approximate hydrostatic balance,

$$
\frac{d \ln p}{d t} \approx-\frac{g w}{R_{d}^{\prime} T}
$$

Substituting Equations (4.1), (4.5) and (4.6) into (4.4) gives

$$
\begin{aligned}
& \frac{d s}{d t}=\frac{L_{f} F_{t}-L_{s} S_{t}+Q-Q_{p}}{T}+c_{l}\left(S_{t}-C_{t}\right) \ln \frac{T}{T_{r e f}}+\left(L_{f} q_{i}-T\left(c_{l} q_{r}+c_{i}\left(q_{s}+q_{g}\right)\right)\right) \\
& \left(L_{f}\left(F_{i}+F_{t}-S_{i}\right)-L_{s} S_{t}+Q-\left(1+\frac{L_{v} q_{v s w}}{R_{d} T}\right) g w\right)\left(\left(C_{p}+C_{p v} q^{*}\right) T^{2}+\frac{L_{v}^{2} q_{v s w}}{R_{v}}\right)^{-1}
\end{aligned}
$$

where the right side represents the source of moist entropy when air is saturated with respect to water. 


\section{b. Sources of moist entropy in a water-unsaturated air parcel}

When an air parcel is unsaturated with respect to water, its energy equation is

$$
\begin{aligned}
& \left(C_{p}+C_{p v} q_{v}+c_{l} q_{r}+c_{i}\left(q_{i}+q_{s}+q_{g}\right)\right) d T-R_{d} T d \ln (p-e)-q_{v} R_{v} T d \ln e \\
& =L_{f} F_{t} d t-L_{v} E_{t} d t-L_{s}\left(S_{t}+S_{i}-F_{i}\right) d t+Q d t-Q_{p} d t
\end{aligned}
$$

where $-F_{i}$ represents the melting of cloud ice to cloud water, and cloud water evaporates immediately once cloud ice melts.

Before deriving the source terms of moist entropy from the preceding equation, some thermal relations are needed for a water-unsaturated parcel. First is an expression for $T_{l}$ the temperature at the lifting condensation level. If a water-unsaturated air parcel is lifted adiabatically to the lifting condensation level, water vapor and energy are conserved. That is

$$
\ln \left(e / E_{s w}\left(T_{l}\right)\right)=\ln \left((p-e) /\left(p_{l}-E_{s w}\left(T_{l}\right)\right)\right)
$$

and

$$
\left(C_{p}+C_{p v} q_{v}\right) \ln \left(T / T_{l}\right)=R_{d} \ln \left((p-e) /\left(p_{l}-E_{s w}\left(T_{l}\right)\right)\right)+R_{v} q_{v} \ln \left(e / E_{s w}\left(T_{l}\right)\right)
$$

where $p_{l}$ is the total air pressure at the lifting condensation level. Eliminating $p_{l}$ in Equation (4.9) yields one equation for $T_{l}$ or Equation (3.6b).

From Equation (3.2), $e$ is represented with $p$ and $q_{v}$ as

$$
\frac{d \ln e}{d t}=\frac{d \ln p}{d t}+\frac{R_{d}}{q_{v}\left(R_{d}+R_{v} q_{v}\right)} \frac{d q_{v}}{d t}
$$

Differentiating Equation (3.6b) and substituting $d T$ into Equation (4.8) yields the tendency of the temperature at the lifting condensation level 


$$
\begin{aligned}
& \frac{d \ln T_{l}}{d t}=\left(\frac{L_{f} F_{t}-L_{v} E_{t}-L_{s}\left(S_{t}+S_{i}-F_{i}\right)+Q-Q_{p}}{T}-C_{p v}\left(q^{*}-q_{v}\right) \frac{d T}{T d t}\right. \\
& \left.-\left(\frac{R_{d}^{2}}{\left(R_{d}+R_{v} q_{v}\right) q_{v}}+\left(R_{v} \frac{C_{p}+C_{p v} q_{v}}{R_{d}+R_{v} q_{v}}-C_{p v}\right) \ln \frac{T}{T_{l}}\right) \frac{d q_{v}}{d t}\right)\left(C_{p}+C_{p v} q_{v}-\frac{R_{d}+R_{v} q_{v}}{R_{v}} \frac{L_{v}\left(T_{l}\right)}{T_{l}}\right)^{-1}
\end{aligned}
$$

with the use of Equations (3.4) and (4.10), where the symbol $q^{*}$ is introduced as

$$
q^{*}=q_{v}+\left(c_{l}\left(q_{t}-q_{v}-q_{i}+q_{r}\right)+c_{i}\left(q_{i}+q_{s}+q_{g}\right)\right) / C_{p v}
$$

The source terms for moist entropy are derived from Equations (3.6) and (4.8). Differentiating Equation (3.6a), then multiplying by $T$ and combining with Equation (4.8) yields

$$
\begin{aligned}
& \frac{d s}{d t}=\frac{L_{f} F_{l}-L_{v}\left(E_{t}+S_{i}-F_{i}\right)-L_{s} S_{t}+Q-Q_{p}}{T}+c_{l} \ln \frac{T}{T_{r e f}} \frac{d q_{t}}{d t} \\
& +q_{v}\left(C_{p v}-c_{l}-\frac{L_{v}\left(T_{l}\right)}{T_{l}}\right) \frac{d \ln T_{l}}{d t}+\left(q_{i} \frac{L_{f}(T)}{T}+c_{l} q_{t}+\left(c_{i}-c_{l}\right) q_{i}-C_{p v} q^{*}\right) \frac{d T}{T d t} \\
& +\left(\frac{L_{v}\left(T_{l}\right)}{T_{l}}+\frac{R_{d} R_{v}}{R_{d}+R_{v} q_{v}}\right) \frac{d q_{v}}{d t}+q_{v} R_{v} \frac{d \ln p}{d t}
\end{aligned}
$$

with the aid of Equations (3.4) and (4.10). Since the terms with $d T / d t$ and $d \operatorname{lnp} / d t$ are small on the right sides of Equations (4.11a) and (4.12), they are approximated using Equation (4.6) and

$$
\frac{d T}{d t} \approx \frac{R_{d} T}{C_{p}+C_{p v} q^{*}} \frac{d \ln p}{d t}+\frac{L_{f} F_{t}-L_{v} E_{t}-L_{s}\left(S_{t}+S_{i}-F_{i}\right)+Q}{C_{p}+C_{p v} q^{*}}
$$

which is obtained from Equation (4.8) after the small terms are ignored. Combining Equations (4.1), (4.2), (4.6), (4.11), (4.12) and (4.13), the source of moist entropy is expressed as 


$$
\begin{aligned}
& \frac{d s}{d t}=\frac{L_{f} F_{t}-L_{s} S_{t}+Q-Q_{p}}{T}+c_{l}\left(E_{t}+S_{t}-C_{t}\right) \ln \frac{T}{T_{\text {ref }}} \\
& +q_{v}\left(C_{p v}-c_{l}-\frac{L_{v}\left(T_{l}\right)}{T_{l}}\right) \lambda_{T l}+\left(q_{i} \frac{L_{f}(T)}{T}+c_{l} q_{t}+\left(c_{i}-c_{l}\right) q_{i}-C_{p v} q^{*}\right) \frac{\lambda_{l}}{T} \\
& +\left(\frac{L_{v}\left(T_{l}\right)}{T_{l}}+\frac{R_{d} R_{v}}{R_{d}+R_{v} q_{v}}\right) S_{t}+\left(\frac{L_{v}\left(T_{l}\right)}{T_{l}}-\frac{L_{v}}{T}+\frac{R_{d} R_{v}}{R_{d}+R_{v} q_{v}}\right)\left(E_{t}+S_{i}-F_{i}\right)-\frac{R_{v} g}{R_{d} T} q_{v} w
\end{aligned}
$$

where the new symbols

$$
\begin{aligned}
& \lambda_{T}=\frac{L_{f} F_{t}-L_{v} E_{t}-L_{s}\left(S_{t}+S_{i}-F_{i}\right)+Q-g w}{C_{p}+C_{p v} q^{*}} \\
& \lambda_{n}=\left(\frac{L_{f} F_{t}-L_{v} E_{t}-L_{s}\left(S_{t}+S_{i}-F_{i}\right)+Q-C_{p v}\left(q^{*}-q_{v}\right) \lambda_{T}}{T}\right. \\
& \left.-\left(\frac{R_{d}^{2}}{\left(R_{d}+R_{v} q_{v}\right) q_{v}}+\left(R_{v} \frac{C_{p}+C_{p v} q_{v}}{R_{d}+R_{v} q_{v}}-C_{p v}\right) \ln \frac{T}{T_{l}}\right)\left(E_{t}+S_{t}+S_{i}-F_{i}\right)\right) \\
& \left(C_{p}+C_{p v} q_{v}-\frac{R_{d}+R_{v} q_{v}}{R_{v}} \frac{L_{v}\left(T_{l}\right)}{T_{l}}\right)^{-1}
\end{aligned}
$$

The approximations in Equations (4.5), (4.6) and (4.13) introduce very small errors in the moist entropy source terms. Scale analysis (omitted) shows that the errors are much smaller than the moist entropy sink from the radiative cooling of $1^{\circ} \mathrm{C} /$ day.

\section{Moist entropy budget}

\section{a. Flux form of the moist entropy equation}

The equation for moist entropy in a precipitating and icy atmosphere is written in a flux form as

$$
\partial_{t} \rho s+\nabla \cdot(\rho \mathbf{v} s)=\rho\left(R_{s}+M_{s}+G_{s}+D_{s}\right)
$$

where $R_{s}, M_{s}, G_{s}$ and $D_{s}$ denote the sources of moist entropy from radiation, microphysics of clouds and precipitation, generation due to mixing, and turbulent diffusion, respectively. The moist entropy radiation term $R_{s}$ is defined as 


$$
R_{s}=Q / T
$$

From Equations (4.7) and (4.14), the microphysics term $M_{s}$ is expressed as

$$
\begin{aligned}
& M_{s}=\frac{L_{f} F_{t}-L_{s} S_{t}-Q_{p}}{T}+c_{l}\left(S_{t}-C_{t}\right) \ln \frac{T}{T_{\text {ref }}}+\left(L_{f} q_{i}-T\left(c_{l} q_{r}+c_{i}\left(q_{s}+q_{g}\right)\right)\right) \\
& \left(L_{f}\left(F_{i}+F_{t}-S_{i}\right)-L_{s} S_{t}+Q-\left(1+\frac{L_{v} q_{v s w}}{R_{d} T}\right) g w\right)\left(\left(C_{p}+C_{p v} q^{*}\right) T^{2}+\frac{L_{v}^{2} q_{v s w}}{R_{v}}\right)^{-1}
\end{aligned}
$$

when air is saturated with respect to water, and

$$
\begin{aligned}
& M_{s}=\frac{L_{f} F_{t}-L_{s} S_{t}-Q_{p}}{T}+c_{l}\left(E_{t}+S_{t}-C_{t}\right) \ln \frac{T}{T_{r e f}} \\
& +q_{v}\left(C_{p v}-c_{l}-\frac{L_{v}\left(T_{l}\right)}{T_{l}}\right) \lambda_{l}+\left(q_{i} \frac{L_{f}(T)}{T}+c_{l} q_{t}+\left(c_{i}-c_{l}\right) q_{i}-C_{p v} q^{*}\right) \frac{\lambda_{T}}{T} \\
& +\left(\frac{L_{v}\left(T_{l}\right)}{T_{l}}+\frac{R_{d} R_{v}}{R_{d}+R_{v} q_{v}}\right) S_{t}+\left(\frac{L_{v}\left(T_{l}\right)}{T_{l}}-\frac{L_{v}}{T}+\frac{R_{d} R_{v}}{R_{d}+R_{v} q_{v}}\right)\left(E_{t}+S_{i}-F_{i}\right)-\frac{R_{v} g}{R_{d} T} q_{v} w
\end{aligned}
$$

when air is unsaturated with respect to water. For simplicity, $M_{s}$ represents a small part of the radiation contribution through $Q$ and $Q$ in $\lambda_{\pi}$.

Turbulence or mixing brings about the diffusion and generation of moist entropy. The diffusion term $D_{s}$ is expressed as

$$
D_{s}=\rho^{-1} \nabla \cdot\left(\rho K_{h} \nabla s\right)
$$

From Equations (2.8) and (B2), the generation term due to mixing $G_{s}$ takes the form

$$
\begin{aligned}
& G_{s}=C_{p}^{-1} K_{h} \nabla s_{d} \cdot \nabla s_{d} \\
& +\frac{K_{h}}{T}\left(c_{l}-c_{i}-\frac{L_{f}(T)}{T}\right) \nabla q_{i} \cdot \nabla T-\frac{K_{h}}{T_{l}}\left(C_{p v}-c_{l}-\frac{L_{v}\left(T_{l}\right)}{T_{l}}\right) \nabla q_{v} \cdot \nabla T_{l} \\
& +\rho^{-1} \nabla \cdot \rho K_{h}\left(\frac{q_{i}}{T}\left(c_{l}-c_{i}-\frac{L_{f}(T)}{T}\right) \nabla T-\frac{q_{v}}{T_{l}}\left(C_{p v}-c_{l}-\frac{L_{v}\left(T_{l}\right)}{T_{l}}\right) \nabla T_{l}\right)
\end{aligned}
$$

\section{b. Surface flux of moist entropy}


The surface flux of moist entropy from the underlying surface into the air above plays an important role in the moist entropy budget. This subsection derives its expression in terms of $Q_{H}$ and $Q_{E}$ the sensible and the latent heat fluxes from the underlying surface to the air.

Consider a thin layer with depth $h$ adjacent to the underlying surface. When air in the layer is saturated with respect to water, the sensible and the latent heat fluxes increase temperature and water content as

$$
\begin{aligned}
& \left(C_{p}+C_{p v} q^{*}\right) \frac{\partial T}{\partial t}-R_{d} T \frac{\partial \ln \left(p-E_{s w}\right)}{\partial t}-q_{v s w} R_{v} T \frac{\partial \ln E_{s w}}{\partial t}=\frac{Q_{H}+Q_{E}}{\rho h} \\
& \frac{\partial q_{t}}{\partial t}=\frac{Q_{E}}{\rho h L_{v}(T)}
\end{aligned}
$$

Since the air is saturated, the mixing ratio of water vapor changes with temperature as

$$
\frac{\partial q_{v s w}}{\partial t}=q_{v s w}\left(\frac{L_{v}(T)}{R_{v} T}-1\right) \frac{\partial T}{T \partial t}
$$

which is derived from Equations (3.2) and (3.4). Differentiating Equation (3.6a) with respect to $T$ and multiplying the resulting equation by $\rho h$ yields the increase of moist entropy in the layer or the surface flux of moist entropy from the underlying surface to the air

$$
\begin{aligned}
& F_{s}=c_{l} \frac{Q_{E}}{L_{v}(T)} \ln \frac{T}{T_{r e f}}+\left(C_{p}+c_{l} q_{t}-R_{d}+q_{v s w}\left(C_{p v}-c_{l}+\left(\frac{L_{v}(T)}{R_{v} T}-2\right) \frac{L_{v}(T)}{T}\right)\right. \\
& \left.-q_{i}\left(c_{l}-c_{i}-\frac{L_{f}(T)}{T}\right)\right) \frac{Q_{H}+Q_{E}}{T\left(C_{p}+C_{p v} q^{*}-R_{d}-q_{v s w} T^{-1} L_{v}(T)\right)}
\end{aligned}
$$

with the use of Equations (5.5) and (5.6).

When air in the layer is unsaturated with respect to water, 


$$
\begin{aligned}
& \left(C_{p}+C_{p v} q^{*}\right) \frac{\partial T}{\partial t}-R_{d} T \frac{\partial \ln (p-e)}{\partial t}-q_{v} R_{v} T \frac{\partial \ln e}{\partial t}=\frac{Q_{H}}{\rho h} \\
& \frac{\partial q_{v}}{\partial t}=\frac{Q_{E}}{\rho h L_{v}(T)}
\end{aligned}
$$

Differentiating Equation (3.6b) with respect to $T$ and making use of Equations (3.2), (3.4) and (5.8), the tendency of temperature at the lifting condensation level is obtained as

$$
\begin{aligned}
& \left(\frac{C_{p}+C_{p v} q_{v}}{R_{d}+R_{v} q_{v}}-\frac{L_{v}\left(T_{l}\right)}{R_{v} T_{l}}\right) \frac{\partial \ln T_{l}}{\partial t}=\left(\left(\frac{C_{p v}}{R_{d}+R_{v} q_{v}}-R_{v} \frac{C_{p}+C_{p v} q_{v}}{\left(R_{d}+R_{v} q_{v}\right)^{2}}\right) \ln \frac{T}{T_{l}}-\frac{1}{q_{v}}\right) \frac{Q_{E}}{\rho h L_{v}(T)} \\
& +\left(\frac{C_{p}+C_{p v} q_{v}}{R_{d}+R_{v} q_{v}}-1\right)\left(\frac{Q_{H}}{\rho h T}+\frac{R_{v} Q_{E}}{\rho h L_{v}(T)}\right)\left(C_{p}+C_{p v} q^{*}-R_{d}-q_{v} R_{v}\right)^{-1}
\end{aligned}
$$

Differentiating Equation (3.6a) with respect to $T$ and then multiplying by $\rho h$ yields the surface flux of moist entropy

$$
\begin{aligned}
& F_{s}=\left(C_{p}+c_{l} q_{t}-R_{d}-q_{i}\left(c_{l}-c_{i}-\frac{L_{f}(T)}{T}\right)\right)\left(\frac{Q_{H}}{T}+\frac{R_{v} Q_{E}}{L_{v}(T)}\right)\left(C_{p}+C_{p v} q^{*}-R_{d}-q_{v} R_{v}\right)^{-1} \\
& +\left(c_{l} \ln \frac{T}{T_{r e f}}+\frac{L_{v}\left(T_{l}\right)}{T_{l}}\right) \frac{Q_{E}}{L_{v}(T)}+q_{v}\left(C_{p v}-c_{l}-\frac{L_{v}\left(T_{l}\right)}{T_{l}}\right)\left(\frac{C_{p}+C_{p v} q_{v}}{R_{d}+R_{v} q_{v}}-\frac{L_{v}\left(T_{l}\right)}{R_{v} T_{l}}\right)^{-1} \\
& \left\{\left(\frac{C_{p}+C_{p v} q_{v}}{R_{d}+R_{v} q_{v}}-1\right)\left(\frac{Q_{H}}{T}+\frac{R_{v} Q_{E}}{L_{v}(T)}\right)\left(C_{p}+C_{p v} q^{*}-R_{d}-q_{v} R_{v}\right)^{-1}\right. \\
& \left.+\left(\left(\frac{C_{p v}}{R_{d}+R_{v} q_{v}}-R_{v} \frac{C_{p}+C_{p v} q_{v}}{\left(R_{d}+R_{v} q_{v}\right)^{2}}\right) \ln \frac{T}{T_{l}}-\frac{1}{q_{v}}\right) \frac{Q_{E}}{L_{v}(T)}\right\}
\end{aligned}
$$

with the use of Equations (5.8) and (5.9).

Equations (5.7) and (5.10) express accurately the surface flux of moist entropy from the underlying surface into the air. They are used, as an example, to calculate the surface flux of moist entropy over ocean. The sensible and the latent heat fluxes over ocean take the following bulk parameterization schemes 


$$
\begin{aligned}
& Q_{H}=\rho C_{p} c_{d} W_{s}\left(T_{s s t}-T\right) \\
& Q_{E}=\rho L_{v} c_{d} W_{s}\left(q_{v s s}-q_{v}\right)
\end{aligned}
$$

where the drag coefficient $c_{d}=1.1 \times 10^{-3} ; W_{s}$ is the surface wind; and $q_{v s s}$ is the saturation mixing ratio at $T_{s s t}$ the sea surface temperature.

The surface flux of moist entropy is usually estimated, for simplicity, with the following approximate formula

$$
F_{\text {sappr }}=\rho c_{d} W_{s}\left(s_{\text {sea }}-s_{\text {air }}\right)
$$

where $s_{s e a}$ and $s_{a i r}$ represent the moist entropy of the surface sea and air, respectively. This approximate formula can be compared with the accurate formula in Equation (5.10) to show its error. Figure 1 displays the error quantities $F_{s}-F_{s a p p r}$ and $\left(F_{s}-F_{s a p p r}\right) / F_{s}$ versus surface relative humidity, when $T_{s s t}=302 \mathrm{~K}$, surface pressure $p=1016 \mathrm{hpa}$, surface air temperature $T=299 \mathrm{~K}$, surface wind $W_{s}=5 \mathrm{~m} \mathrm{~s}^{-1}$, and there is neither ice nor precipitating particles. Figure 2 is the same as Figure 1 except that the air surface moist entropy $s_{a i r}=230 \mathrm{~J} \mathrm{~kg}^{-1} \mathrm{~K}^{-1}$ instead of $T$ is fixed. As both figures show, the surface flux of moist entropy in Equation (5.12) contains about $2 \%$ relative error that is equivalent to a moist entropy sink from atmospheric cooling of $0.04^{\circ} \mathrm{C} /$ day. Such error, as shown in the next subsection, can distort the modeling of large-scale vertical circulations in the tropics.

\section{c. The moist entropy budget in a tropical atmosphere}

Using the equation for moist entropy, this subsection analyzes the moist entropy budget in a tropical oceanic atmosphere to show the necessity for an accurate surface moist entropy flux in modeling large-scale vertical circulations. Neelin and Held (1987) constructed a simple model on the moist static energy budget, providing a starting point 
for understanding large-scale vertical circulations in the tropics. In contrast to moist static energy, moist entropy is a conserved variable for an air parcel in adiabatic motion. Thus, their model is rewritten in moist entropy so as to incorporate a parcel dynamics methodology.

The internal source of moist entropy is very small in contrast to $F_{r}$, the radiative flux of moist entropy at the tropopause, and $F_{s}$, the surface flux of moist entropy from the ocean into the air above (Zeng 2001). Thus, in a quasi-steady atmosphere, the inflow of moist entropy is balanced by the outflow of moist entropy, or

$$
F_{s}-F_{r}=\int_{p_{t}}^{p_{s}} s \nabla \cdot \mathbf{v}_{h} g^{-1} d p
$$

where the horizontal difference in moist entropy $\mathbf{v}_{h} \cdot \nabla s$ is ignored (Neelin and Held 1987). Just as Equation (5.13) states and Figure 3 shows schematically, the increase in moist entropy through the bottom of the atmosphere and the top must be balanced through the lateral boundary by the large-scale vertical circulation. Hence the large-scale vertical circulation is controlled by $F_{s}-F_{r}$ with the aid of the mass continuity equation

$$
\int_{p_{t}}^{p_{s}} \nabla \cdot v_{h} g^{-1} d p=0
$$

The model in Equations (5.13) and (5.14) is applied to the atmosphere with the average tropical profile (Jordan 1958) to diagnose large-scale vertical velocity. The average profile is displayed on the left side of Figure 3. Thick and thin solid lines represent moist entropy and saturation moist entropy versus height, respectively. Points A and B indicate surface moist entropy and saturation moist entropy, respectively. Thick and thin dashed lines represent the moist entropy and saturation moist entropy of a lifted surface air parcel, respectively. When the surface air parcel moves upward, it passes the 
lifting condensation level (LCL) first, then the level of free convection (LFC), and finally reaches Point $C$ with zero buoyancy. Point $C$ usually corresponds to the tropopause. Let $p_{t}$ represent the pressure at Point $\mathrm{C}$. Thus large-scale vertical circulations are confined below the height $p_{t}$.

Figure 3 shows that moist entropy decreases with height at first, and then increases with height. The vertical profile of moist entropy is fitted with the following function

$$
s= \begin{cases}s_{\min }+\frac{s_{s}-s_{\min }}{p_{s}-p_{\text {min }}}\left(p-p_{s \min }\right) & p_{s \min }<p<p_{s} \\ s_{\min }+\frac{s_{t}-s_{\min }}{p_{t}-p_{s \min }}\left(p-p_{s \min }\right) & p_{t}<p<p_{s \min }\end{cases}
$$

where $p_{s}$ is surface pressure, and $p_{s \min } \approx 700 \mathrm{hpa}$ is the pressure with the minimum moist entropy $s_{\min }$. In the atmosphere with the preceding vertical distribution of moist entropy, small cumulus clouds and large turbulent moist eddies lead to the down-gradient transport of moist entropy between $p_{s}$ and $p_{\text {smin }}$, and deep convective clouds lead to the up-gradient transport of moist entropy between $p_{s \min }$ and $p_{t}$ (Riehl and Malkus 1958). With buoyancy sorting (Raymond and Blyth 1986), cumulus clouds lead to a large-scale vertical velocity with a maximum value at the level $p_{w \max }$ higher than $p_{s \min }$, which corresponds to the concept of "gross moist stability" that Neelin and Held (1987) proposed. Thus it is assumed that the vertical distribution of horizontal divergence

$$
\nabla \cdot \mathbf{v}_{h}= \begin{cases}\frac{p-p_{w \max }}{p_{s}-p_{w \max }} \nabla \cdot \mathbf{v}_{h s} & p_{w \max }<p<p_{s} \\ \frac{p-p_{w \max }}{\left(p_{t}-p_{w \max }\right)^{2}}\left(p_{s}-p_{w \max }\right) \nabla \cdot \mathbf{v}_{h s} & p_{t}<p<p_{w \max }\end{cases}
$$

where $\nabla \cdot \mathbf{v}_{h s}$ is surface horizontal divergence and $p_{w \max } \approx 650 \mathrm{hpa}$ is estimated. 
Equations (5.15) and (5.16) determine the net lateral output of moist entropy that is caused by the large-scale vertical circulation. Substituting Equations (5.15) and (5.16) into (5.13) yields a solution of the surface horizontal divergence $\nabla \cdot v_{h s}$. Then the vertical distribution of large-scale vertical velocity is obtained as a function of $F_{s}-F_{r}, s_{\min }, p_{s \min }$ and $p_{w \max }$.

In the model in Equations (5.15) and (5.16), the large-scale vertical circulation is controlled by $F_{s}-F_{r}$. Since the tropical atmosphere stays close to radiative-convective equilibrium, $F_{s}$ is close to $F_{r}$. Therefore $\left(F_{s}-F_{r}\right) / F_{s}$ is very small. If the formula for the surface flux of moist entropy such as Equation (5.12) contains $\sim 2 \%$ relative error, it distorts the modeling of the large-scale vertical circulation. Summarily, Equations (5.7) and (5.10) express the surface flux of moist entropy accurately. They are therefore needed for long-term cloud modeling, where large-scale vertical circulations are involved.

\section{Summary and future work}

This theoretical paper deals with the equation for moist entropy in a precipitating and icy atmosphere. On the basis of the consistency between the energy and the entropy equations, the paper derives accurate source terms for moist entropy from the energy equation, including those in relation to the microphysics of clouds and precipitation. As a result, the paper obtains a complete equation for moist entropy with explicit internal and external sources. Since the equation is equivalent to energy equation, it provides a basis for using moist entropy as a prognostic variable in the next generation of cloud-resolving models (e.g., a global cloud-resolving model). 
In addition, the paper derives an accurate formula for the surface flux of most entropy from the underlying surface into the air above. Such an accurate formula is necessary in long-term cloud modeling, because the tropical large-scale vertical velocity is sensitive to the surface flux of moist entropy.

Moist entropy is nearly conserved in adiabatic motion. It is redistributed rather than created by moist convection. Thus an accurate equation for moist entropy, as a healthy direction, can be used to construct analytical and numerical models of moist convection. In this approach for the next generation of cloud-resolving models, moist entropy will be used as a prognostic variable, and this paper provides a theoretical basis. In addition to this work on entropy budget, other numerical techniques need to be developed to treat sound waves and diagnose the temperature for correct energy and entropy conversion. Those numerical techniques will be introduced elsewhere.

Analytical models of moist convection written in moist entropy, just as that in Section 5.c, are usually based on the assumption that the internal sink of moist entropy is small enough to be ignored. Zeng (2001) used a three-dimensional numerical model of warm clouds to show that the internal sink of moist entropy is very small in contrast to the moist entropy sink from the atmospheric cooling of $1^{\circ} \mathrm{C} /$ day. Since ice plays a role in some atmospheric phenomena, the assumption needs to be re-checked when ice is involved. This paper provides an expression for the internal sink of moist entropy. The expression will be used to estimate the magnitude of the internal sink of moist entropy with the aid of cloud-resolving modeling. 
Acknowledgements. The work is supported by the NASA Headquarters Atmospheric Dynamics and Thermodynamics Program and the NASA Tropical Rainfall Measuring Mission (TRMM). The authors are grateful to Dr. R. Kakar at NASA headquarters for his support of this research. The authors also thank Mr. S. Lang for reading the manuscript. 


\section{APPENDIX A}

\section{List of Symbols}

$C_{p} / C_{p v} / c / c_{i}:$ specific heat of dry air/water vapor/liquid water/ice

$C_{t}:$ total conversion rate from cloud water and ice to precipitation

$D_{s}$ : subgrid diffusion of moist entropy due to mixing, see (5.4a)

$e:$ partial pressure of water vapor

$E_{s w}:$ saturation vapor pressure over water

$E_{\Downarrow} / F_{\downarrow} / S_{t}$ : total rate of evaporation/freezing/sublimation of precipitating particles

$F_{i}:$ rate of cloud water freezing or cloud ice melting

$F_{r}$ : radiative flux of moist entropy at the tropopause

$F_{s}:$ surface flux of moist entropy from the underlying surface into the air above, see (5.7)

and (5.10)

$g:$ acceleration due to gravity

$G_{s}:$ generation of moist entropy due to mixing, see (5.4b)

$K_{h}$ : eddy mixing coefficient for scalars

$L_{\checkmark} / L_{\S} / L_{f}$ : latent heat of vaporization/sublimation/freezing, see (3.1)

$M_{s}$ : microphysical source of moist entropy, see (5.3)

$p:$ total pressure of moist air

$p_{\text {ref }}=10^{5}$ pa : reference pressure

$q$ : heat absorbed by a unit mass of air

$q^{*}:$ defined in $(4.11 b)$

$q_{v} / q_{d} / q_{i} / q_{r} / q_{s} / q_{g}:$ mixing ratio of water vapor/cloud water/cloud

ice/rainwater/snow/graupel 
$q_{v s w}:$ saturation mixing ratio of water vapor over water

$q_{r}=q_{v}+q_{c}+q_{i}:$ total mixing ratio of airborne water

$Q:$ rate of diabatic heating

$Q_{H} / Q_{E}$ : sensible/latent heat flux from the underlying surface into the air above

$Q_{p}$ : heat transfer from air to precipitating particles, see (C1)

$R_{d} / R_{v}$ : gas constant of dry air/water vapor

$R_{s}:$ radiative source of moist entropy, see (5.2)

$s:$ (moist) entropy of a unit mass, defined in (3.6)

$s_{d}$ : entropy of dry air, see (2.6)

$S_{i}$ : sublimation of cloud ice or deposition of water vapor on cloud ice

$t:$ time

$T:$ temperature

$T_{l}:$ air temperature at the lifting condensation level, defined in (3.6b)

$T_{\text {ref }}=273.15 \mathrm{~K}:$ reference temperature

$\mathbf{v}$ : velocity vector

$\mathbf{v}_{h}$ : horizontal velocity

$V_{r} / V_{s} / V_{g}:$ fall speeds of rain/snow/graupel

$w$ : velocity velocity

$z:$ height

$\rho:$ air density

$\lambda_{T} / \lambda_{T l}:$ defined in (4.15) 


\section{APPENDIX B}

\section{Irreversible Generation of Moist Entropy due to Mixing}

Section 2 derives the irreversible generation of dry entropy due to mixing. This appendix discusses the irreversible generation of moist entropy due to mixing. For a moist atmosphere, there are the following two equations

$$
\begin{aligned}
& \rho \frac{d q_{v}}{d t}+\cdots=\nabla \cdot\left(\rho K_{h} \nabla q_{v}\right) \\
& \rho \frac{d q_{i}}{d t}+\cdots=\nabla \cdot\left(\rho K_{h} \nabla q_{i}\right)
\end{aligned}
$$

where the right sides represent the effects of mixing. Multiplying Equation (B1a) by $L_{\nu}\left(T_{l}\right) / T_{l}$, subtracting (B1b) times $L_{f}(T) / T$, and rearranging terms yields

$$
\begin{aligned}
& \rho \frac{L_{v}\left(T_{l}\right)}{T_{l}} \frac{d q_{v}}{d t}-\rho \frac{L_{f}(T)}{T} \frac{d q_{i}}{d t}+\cdots=\nabla \cdot\left(\rho K_{h} \nabla\left(\frac{L_{v}\left(T_{l}\right)}{T_{l}} q_{v}-\frac{L_{f}(T)}{T} q_{i}\right)\right) \\
& +\frac{\rho K_{h}}{T}\left(c_{l}-c_{i}-\frac{L_{f}(T)}{T}\right) \nabla q_{i} \cdot \nabla T-\frac{\rho K_{h}}{T_{l}}\left(C_{p v}-c_{l}-\frac{L_{v}\left(T_{l}\right)}{T_{l}}\right) \nabla q_{v} \cdot \nabla T_{l} \\
& +\nabla \cdot \rho K_{h}\left(\frac{q_{i}}{T}\left(c_{l}-c_{i}-\frac{L_{f}(T)}{T}\right) \nabla T-\frac{q_{v}}{T_{l}}\left(C_{p v}-c_{l}-\frac{L_{v}\left(T_{l}\right)}{T_{l}}\right) \nabla T_{l}\right)
\end{aligned}
$$

with the use of Equation (3.1). On the right side of the above equation, the last two terms represent the irreversible generation of moist entropy due to mixing except for the last term in Equation (2.8). 


\section{APPENDIX C \\ Energy Transfer from Air to Precipitating Particles}

Precipitating particles fall with respect to air. As a result, they move downward from one parcel to another parcel. Since their temperature is usually lower than the atmospheric temperature, heat is transferred from the air to the precipitating particles. This appendix derives an expression for the heat transfer from air to precipitating particles.

In a Cartesian coordinate system moving with the air, a cell with unit horizontal area spans the height $z$ to $z+\Delta z$. Precipitating particles fall into the cell at the top with the flux $\rho(z+\Delta z) q_{j}(z+\Delta z) V_{j}$, where $V_{j}$ denotes the terminal velocity of precipitating particles. Assume that the particles distribute uniformly in the cell and keep the same temperature as the air. Thus, the particles change their temperature from $T(z+\Delta z)$ to $T(z)$. As a result, the precipitating particles absorb energy from the air at the rate $c_{j} \rho q_{j} V_{j}(T(z+\Delta z / 2)-T(z+\Delta z)) / \Delta z$ or $-\frac{1}{2} c_{j} \rho q_{j} V_{j} \frac{\partial T}{\partial z}$ in the unit volume, where $c_{j}$ is the specific heat of either water or ice.

Precipitating particles are rainwater, snow and graupel/hail. Thus the heat transfer from air to precipitating particles in unit mass of dry air is expressed as

$$
Q_{p}=-\frac{1}{2}\left(c_{l} q_{r} V_{r}+c_{i} q_{s} V_{s}+c_{i} q_{g} V_{g}\right) \frac{\partial T}{\partial z}
$$

In other words, precipitating particles bring about energy exchange between air parcels. 


\section{REFERENCES}

Betts, A. K., 1973: Non-precipitating cumulus convection and its parameterization. Quart. J. Roy. Meteor. Soc., 99, 178-196.

Charney, J. G. and A. Eliassen, 1964: On the growth of the hurricane depression. $J$. Atmos. Sci., 21, 68-75.

Emanuel, K. A., 1994: Atmospheric Convection. Oxford University Press, New York, 580pp.

Emanuel, K. A., 1995: The behavior of a simple hurricane model using a convective scheme based on subcloud-layer entropy equilibrium. J. Atmos. Sci., 52, 39603968.

Grabowski, W. W., 1989: Numerical experiments on the dynamics of the cloudenvironment interface: small cumulus in a shear-free environment. J. Atmos. Sci., 46, 3513-3541.

Hauf, T. and H. Höller, 1987: Entropy and potential temperature. J. Atmos. Sci., 44, 2887-2901.

Iribarne, J. V. and W. L. Godson, 1981: Atmospheric Thermodynamics. 2nd Ed. Reidel, $259 \mathrm{pp}$.

Jordan, C. L., 1958: Mean sounding for the west Indies area. J. Meteor., 15, 91-97.

Morse, P. M., 1969: Thermal Physics. 2nd Ed. W. A. Benjamin, 431 pp.

Neelin, J. D. and I. M. Held, 1987: Modeling tropical convergence based on the moist static energy budget. Mon. Wea. Rev., 115, 3-12.

Ooyama, K. V., 1990: A thermodynamic foundation for modeling the moist atmosphere. J. Atmos. Sci., 47, 2580-2593. 
Ooyama, K. V., 2001: A dynamic and thermodynamic foundation for modeling the moist atmosphere with parameterized microphysics. J. Atmos. Sci., 58, 2073-2102.

Pruppacher, H. R. and J. D. Klett, 1978: Microphysics of clouds and precipitation. Reidel, $714 \mathrm{pp}$.

Riehl, H. and J. S. Malkus, 1958: On the heat balance in the equatorial trough zone. Geophysica, 6, 503-537.

Raymond, D. J., 1995: Regulation of moist convection over the west Pacific warm pool. J. Atmos. Sci., 52, 3945-3959.

Raymond, D. J., 2000: Thermodynamic control of tropical rainfall. Quart. J. Roy. Meteor. Soc., 126, 889-898.

Raymond, D. J. and A. M. Blyth, 1986: A stochastic mixing model for nonprecipitating cumulus clouds. J. Atmos. Sci., 43, 2708-2718.

Raymond, D. J. and X. Zeng, 2000: Instability and large scale circulations in a twocolumn model of the troposphere. Quart. J. Royal Meteor. Soc., 126, 3117-3135.

Satoh, M., 2002: Conservative scheme for the compressible nonhydrostatic models with the horizontally explicit and vertically implicit time integration scheme. Mon. Wea. Rev., 130, 1227-1245.

Tao, W.-K. and J. Simpson, 1993: The Goddard Cumulus Ensemble model. Part I: Model description. Terr. Atmos. Ocean Sci., 4, 19-54.

Tao, W.-K., J. Simpson and M. McCumber, 1989: An ice-water saturation adjustment. Mon. Wea. Rev., 117, 232-235.

Tao, W.-K., J. Simpson, D. Baker, S. Braun, M.-D. Chou, B. Ferrier, D. Johnson, A. Khain, S. Lang, B. Lynn, C.-L. Shie, D. Starr, C.-H. Sui, Y. Wang and P. Wetzel, 
2003: Microphysics, radiation and surface processes in the Goddard Cumulus Ensemble (GCE) model. Meteor. Atmos. Phys., 82, 97-137.

Tompkins A. M. and G. C. Craig, 1998: Radiative-convective equilibrium in a threedimensional cloud ensemble model. Quart. J. Roy. Meteor. Soc., 124, 2073-2098.

Wallace, J. M. and P. V. Hobbs, 1977: Atmospheric Science: An Introductory Survey. Academic Press, New York, 467pp.

Zeng, X., 2001: Ensemble simulation of tropical convection. Ph.D. dissertation, New Mexico Tech. New Mexico Tech Library, 124pp. 


\section{Figure Captions}

Figure 1 Error in the surface flux of moist entropy in Equation (5.12) with respect to surface relative humidity when the air surface temperature $T=299 \mathrm{~K}$ is fixed.

Figure 2 the same as Figure 1 except that the air surface moist entropy $s_{a i r}=230 \mathrm{~J} \mathrm{~kg}^{-1} \mathrm{~K}^{-}$ ${ }^{1}$ is fixed.

Figure 3 Vertical distribution of moist entropy (thick solid line) and saturation moist entropy (thin solid line) and a schematic of the large-scale vertical circulation in the Tropics. Points A and B indicate surface moist entropy and saturation moist entropy, respectively. Thick and thin dashed lines represent moist entropy (Line A-LFC-C) and saturation moist entropy (Line B-LCL-C) of a lifted surface parcel, respectively. 

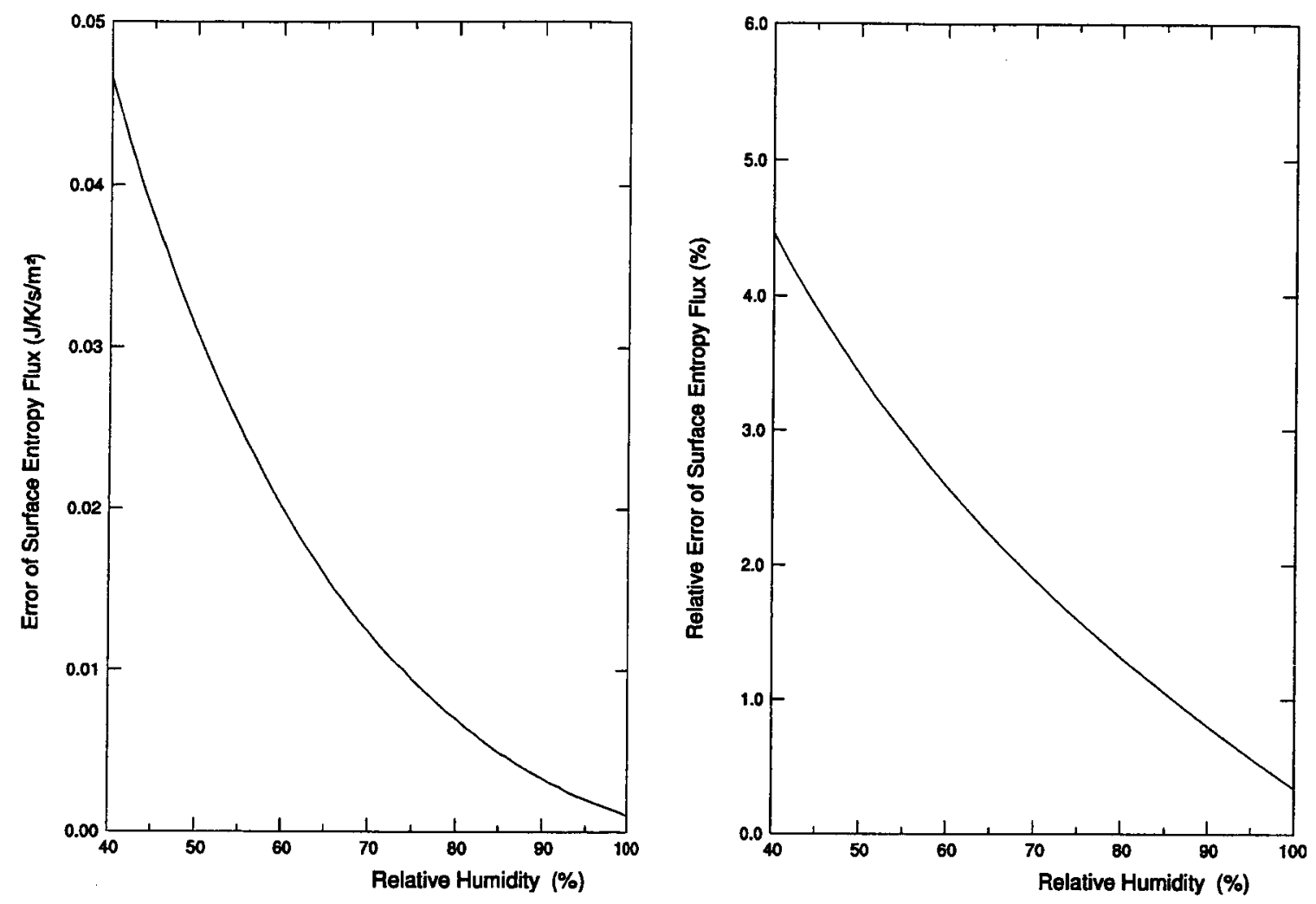

Figure 1 Error in the surface flux of moist entropy in Equation (5.12) with respect to surface relative humidity when the air surface temperature $T=299 \mathrm{~K}$ is fixed. 

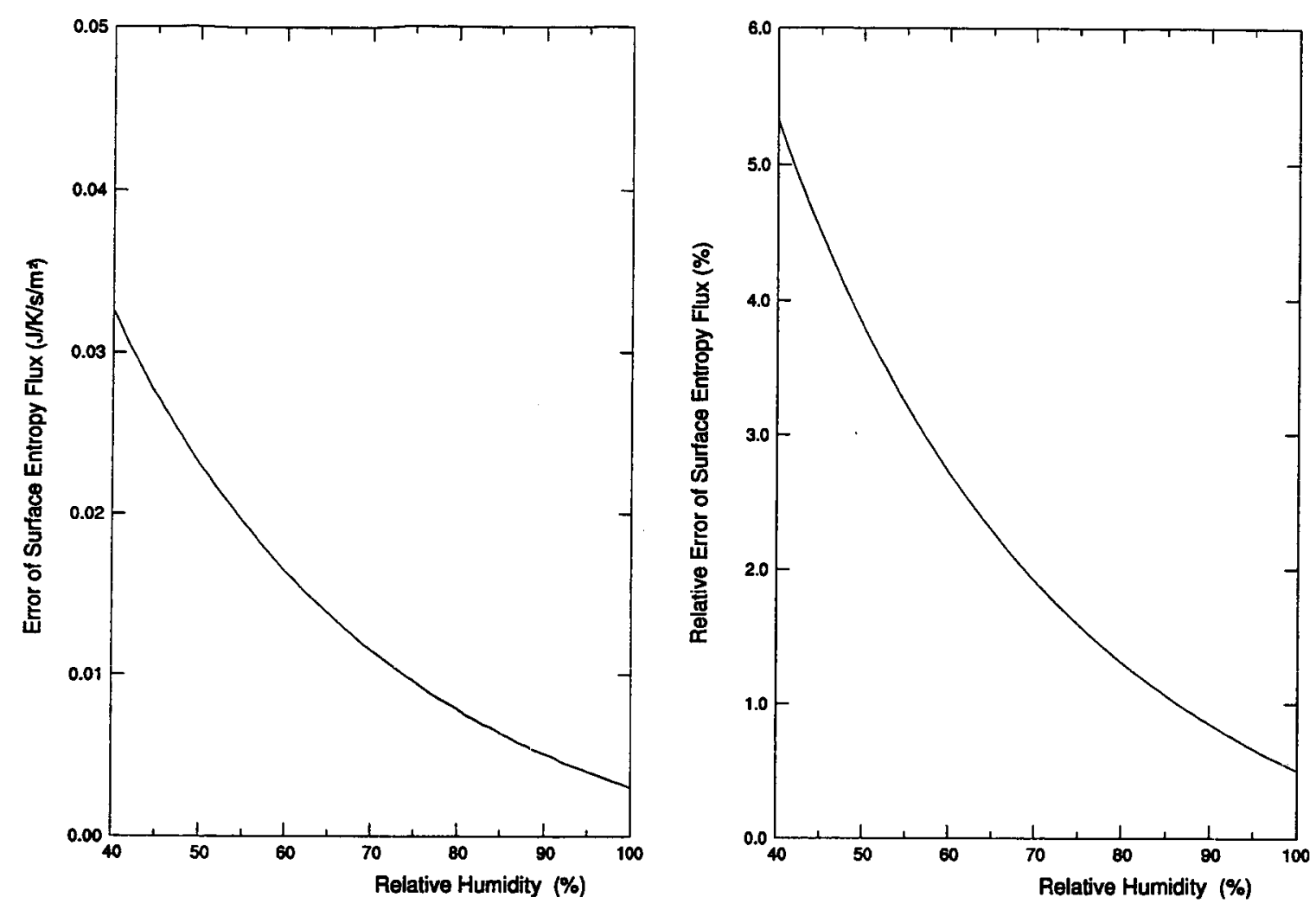

Figure 2 the same as Figure 1 except that the air surface moist entropy $s_{a i r}=230 \mathrm{~J} \mathrm{~kg}^{-1} \mathrm{~K}^{-1}$ is fixed. 

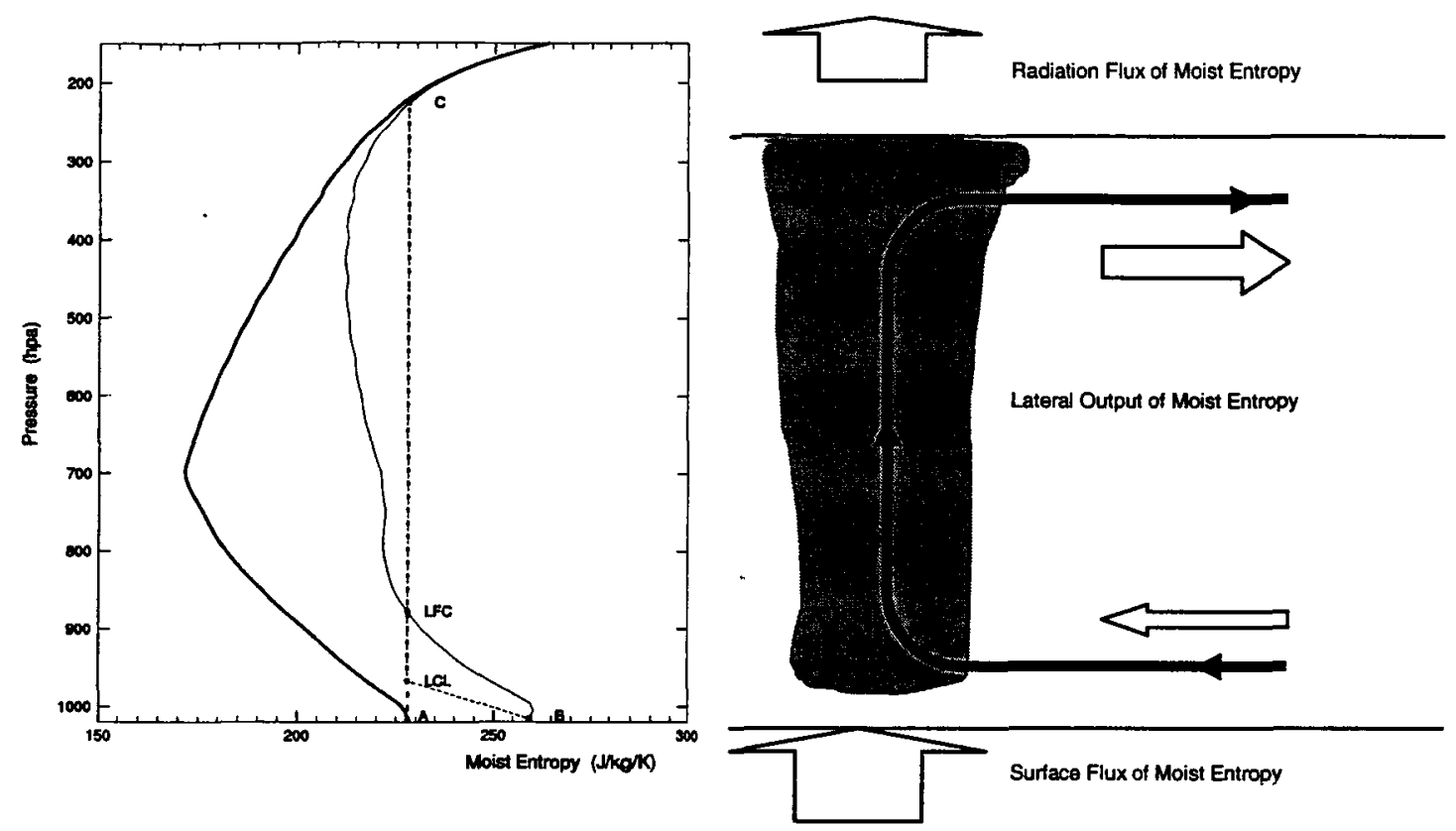

Figure 3 Vertical distribution of moist entropy (thick solid line) and saturation moist entropy (thin solid line) and a schematic of the large-scale vertical circulation in the Tropics. Points A and B indicate surface moist entropy and saturation moist entropy, respectively. Thick and thin dashed lines represent moist entropy (Line A-LFC-C) and saturation moist entropy (Line B-LCL-C) of a lifted surface parcel, respectively. 